\title{
Breast Cancer and Novel Therapeutic Treatments
}

\author{
a report by \\ John R Benson \\ Consultant Breast Surgeon, Cambridge Breast Unit, Addenbrooke's Hospital, Cambridge
}

DOI: $10.17925 / E O H \cdot 2007.0 .2 .47$

Breast cancer remains the most common malignancy among women, with an average lifetime risk of approximately $10 \%$. Despite the continued rise in incidence of the disease, with almost half a million deaths annually worldwide, mortality rates have fallen over the past two decades. This is testimony to the success of interventional strategies such as screening and adjuvant systemic therapies that permit diagnosis of breast cancer prior to de novo formation of micrometastases or the obliteration of established foci of disease at distant sites. It is this burden of micrometastatic disease outside the breast that represents the most fundamental and challenging aspect of breast cancer treatment.

\section{Biological Models}

In accordance with Fisher's hypothesis of biological predeterminism, these micrometastatic foci can remain dormant and be activated many years after initial diagnosis. ${ }^{1}$ It is now acknowledged that not all cases of early breast cancer are systemic at the outset with distant micrometastases pre-existent at presentation. Breast cancer is a heterogeneous disease with a variable and unpredictable natural history. We have entered a new era in breast cancer management where disease is 'small' and more likely to be confined to the breast and regional nodes. ${ }^{2}$ This 'stage migration' is attributable to a combination of heightened public awareness and screening programmes and has led to an increased proportion of smaller-sized $(<2 \mathrm{~cm})$ node-negative tumours. Some of these tumours will behave in a Halstedian manner with minimal proclivity for haematogenous dissemination and the formation of micrometastases at an early stage in the neoplastic continuum. A spectrum, or intermediate paradigm, is emerging that encompasses elements of Fisher and Halsted but is less restrictive than either hypothesis in pure form. ${ }^{3}$

Modern methods of molecular profiling may permit tumours to be assigned to one group or another based on biological behaviour, with appropriate intensities of locoregional and systemic treatments. Those patients without micrometastatic disease at presentation do not require adjuvant systemic therapy and fewer than $10 \%$ of those receiving chemotherapy for node-negative disease derive any benefit. It may be surmised that as tumour size has fallen progressively in recent years, a lower proportion of patients will have micrometastases at the time of diagnosis and a correspondingly greater proportion will have disease limited to the locoregional tissues. For these patients, inadequate primary locoregional treatment will lead to higher rates of local recurrence, which under these circumstances represents a determinant of distant disease and can directly affect survival by acting as a source for micrometastases. ${ }^{4}$ The biphasic pattern of recurrence with peaks at one to two and four to five years suggests that dormant micrometastases may be stimulated by the act of primary surgery, which can remove sources of angiostatin with initiation of microangiogenesis and the dissemination of tumour cells.
Administration of antiangiogenic agents in a pre-surgical schedule may suppress this angiogenic kick-start and interrupt the 'conversation' between breast cancer and endothelial cells. ${ }^{5}$

\section{Locoregional Treatment}

The latest overview by the Early Breast Cancer Trialists Collaborative Group (EBCTCG) has confirmed an overall survival benefit at 15 years from local radiation treatment to either the breast, following breast conservation therapy, or the chest wall, after mastectomy. The absolute reductions in local recurrence at five years and mortality at 15 years are 19 and 5\%, respectively. ${ }^{6}$ This represents one life saved for every four local recurrences prevented by radiotherapy at five years. Alhough these survival benefits are relatively modest, they emphasise the importance of surgery and radiotherapy in the avoidance of persistent disease and relapse in locoregional tissues that can act as a source for micrometastases and directly impair long-term survival. There is a risk that minimally effective treatments may compromise locoregional control in some patients. It is essential that all forms of breast conservation surgery achieve histologically negative margins and that radiobiological equivalence to conventional external beam therapy is demonstrable for newer techniques of breast irradiation such as intra-operative, partial-breast and intensity-modulated radiotherapy.

Skin-sparing mastectomy (SSM) represents the latest phase in the development of progressively less mutilating forms of mastectomy for breast cancer treatment. ${ }^{7}$ The oncological equivalence of SSM to standard forms of modified radical mastectomy has never been validated in prospective controlled trials. There is a potential risk of increased local recurrence when peri-areolar incisions are adopted in a blanket manner or when general (breast) surgeons are coerced into performing 'pure' skin-sparing resections when these are inappropriate. ${ }^{8}$ Long-term data on rates of locoregional and distant recurrence will clarify relative indications for SSM, but, in the interim, selection criteria and quality control issues must be monitored and subjected to ongoing audit and evaluation. ${ }^{9} \mathrm{~A}$ small group of patients may be suited to an oncoplastic technique in which a relatively large resection of breast tissue is performed with

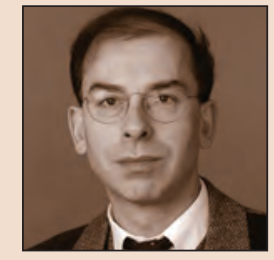

John R Benson is a Consultant Breast Surgeon at Addenbrooke's Hospital and Director of Clinical Studies at Selwyn College, Cambridge. He has written over 70 manuscripts and is the co-author of two best-selling textbooks in the fields of breast and reconstructive surgery. He qualified from Oxford University Clinical School and received specialist training in breast diseases at the Royal Marsden Hospital and the Institute of Cancer Research, London, UK. Further experience was obtained as Visiting Professor at the New York Hospital-Cornell Medical Center, New York.

E: john.benson@addenbrookes.nhs.uk 


\section{Breast Cancer}

Figure 1: Sentinel Lymph Node Highlighted by Blue Dye

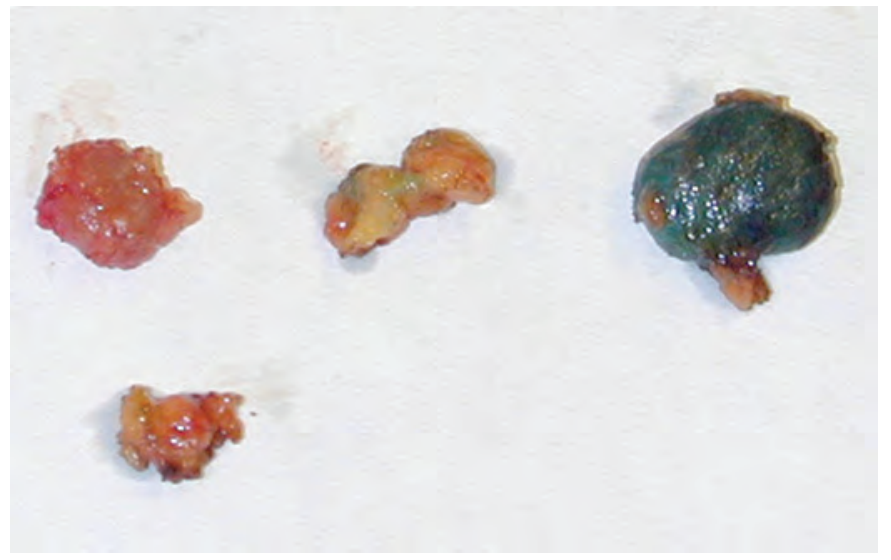

Figure 2: Digital Mammogram Machine

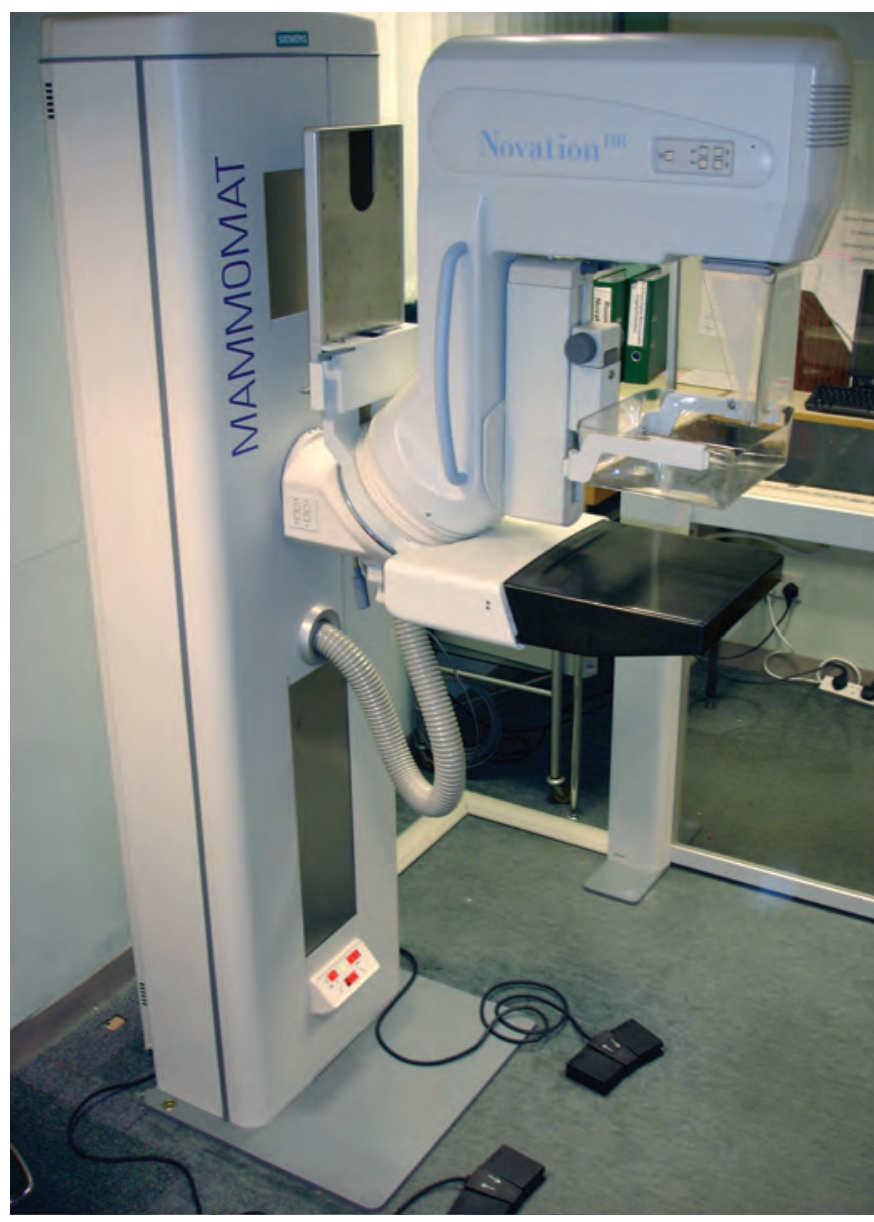

subsequent transposition of remaining breast parenchyma to re-shape the breast. In the event of margin positivity, further re-excision can be difficult and a complete mastectomy may be necessary. The majority of patients are best managed with either standard breast conservation (wide local excision) or SSM with immediate breast reconstruction.

\section{Sentinel Node Biopsy}

The technique of sentinel node biopsy is now widely practised in many centres around the world and has become a standard of care. This minimalist approach to axillary management has relevance to earlier-stage disease, where node negativity is more common. A review by the American Society of Clinical Oncology (ASCO) Technology Assessment panel reaffirmed that dual localisation techniques with a combination of blue dye and isotope maximises identification rates (>90\%) and are associated with high negative predictive values (>95\%) with a short learning curve (see Figure 1). ${ }^{10}$ Overall false-negative rates are between 5 and 10\% (mean $8.4 \%$ ) and are minimised by intra-operative digital examination and the removal of nodes that are suspicious but neither hot nor blue (see Figure 2). This 'sentinel plus' technique has merged with blue-dye-assisted node sampling, which is popular in the UK and may be the most pragmatic approach when nuclear medicine facilities are limited. ${ }^{11}$ Removal of three to four nodes accords with anatomical patterns of lymphatic drainage to the axillary basin. ${ }^{12}$ There is a lack of consensus as to whether some patients with smaller invasive tumours can avoid sentinel node biopsy and, conversely, whether this technique is less accurate for tumours $>3 \mathrm{~cm}$. Preoperative axillary ultrasound and core biopsy is likely to select a high proportion of node-positive patients and intra-operative node examination may reduce numbers of patients requiring a delayed axillary procedure..$^{13}$ There are persistent uncertainties about the significance of micrometastatic deposits within the sentinel node(s), especially when only a single node is involved. More intense scrutiny of nodal tissue with immunohistochemistry/ PCR potentially upstages $20-30 \%$ of patients, with risk of overtreatment. Consequences of the failure to remove non-sentinel nodes remain unknown, although rates of axillary relapse following sentinel node biopsy are very low ( $2 \%$ at three years) and these are unlikely to translate into any meaningful reduction in long-term survival. ${ }^{14}$ Ongoing trials will determine whether completion axillary dissection is mandatory in all sentinel nodepositive patients and could be omitted in some cases without detriment to locoregional control or overall survival. ${ }^{15,16} \mathrm{~A}$ range of options for axillary management based on risk/cost-benefit analyses, together with patient choice, will probably prove to be the ideal practice. ${ }^{17}$

\section{Systemic Treatments}

It is the presence of distant micrometastases at presentation that ultimately determines a patient's clinical fate. Systemic therapies target these microscopic foci of tumour and their modes of action are increasingly based on an understanding of the biological events underlying disordered growth patterns. The microenvironment of a tumour contains a pool of growth factors, which may be stimulatory or inhibitory to cell growth. These form a component of the complex language of intercellular communication that is often disrupted in the malignant state, leading to the autonomous growth of cells. Some channels of communication persist and cancer cells can be tamed or re-regulated. This concept of 'cell control' rather than 'cell kill' exploits the similarities between cancer and normal cells. ${ }^{18}$ Chemotherapy schedules based on cytotoxicity are unlikely to modulate levels of growth factors in any consistent and meaningful manner. Rather than selectively enhancing or suppressing cellular functions, the primary action of chemotherapeutic agents is to indiscriminately kill cancer cells by dislocating biochemical pathways, interfering with DNA repair processes and inducing 'cell suicide' (apoptosis). The newer biological response modifiers exploit these natural growth regulatory mechanisms and target mitogenic pathways at various levels: ligand, growth factor receptor or post-receptor signal transduction.

\section{Biological Response Modifiers}

These translational approaches are exemplified by trastuzumab or Herceptin, a humanised recombinant monoclonal antibody that targets the human epidermal growth factor receptor 2 (HER2). This is expressed in normal and malignant breast epithelial cells, but overexpressed in $20-30 \%$ of all breast cancers. In pre-clinical studies, overexpression of 
the HER2/neu oncogene results in increased rates of proliferation, loss of contact inhibition, enhanced growth in soft agar and a more tumorigenic phenotype in vivo with greater metastatic potential. Recent trials have shown that the use of Herceptin for one to two years, following standard chemotherapy regimens in HER2-positive patients, prolongs disease-free survival and reduces rates of relapse by up to $50 \% .{ }^{19}$ Inhibition of HER2 overexpression can restore or increase oestrogen receptor (OR) expression, thus overcoming endocrine resistance and allowing further hormonal manipulation. ${ }^{20} \mathrm{~A}$ potential novel target related to HER2 is the protein GP88, which is expressed in invasive ductal carcinoma but not in benign tissue (see Figure 3). Laboratory models suggest that this can constitutively activate HER2 by phosphorylation and prevent subsequent inhibition by Herceptin. ${ }^{21}$ Dual targeting of HER2 and GP88 may prove to be more clinically effective than the use of Herceptin as a single agent. The relative magnitude of gains from Herceptin is similar across all subgroups and there is less than a $20 \%$ chance that benefit will be lost with more prolonged follow-up. There is compound cardiotoxicity from combined therapy with Herceptin and taxanes that should be sequenced when baseline left ventricular ejection factor (LVEF) is suboptimal. The enzyme topoisomerase 2 is amplified in 35\% of HER2-positive patients and a fluorescence in situ hybridisation (FISH) assay for this enzyme may help identify those patients most likely to respond to taxanes and Herceptin.

Tumour growth is dependent on new blood vessel formation, and inhibitors of angiogenesis are entering clinical trials as a combined adjuvant. Xenograft tumour models have shown marked reductions in tumour growth when trastuzumab is administered concomitantly with antivascular endothelial growth factor (anti-VEGF) antibodies. A phase I trial of trastuzumab and the antiangiogenic agent Avastin (bevacizumab) has confirmed this combination to be clinically safe and effective. A phase II study is currently under way to further evaluate this combination. Avastin is also being incorporated into standard regimens of anthracyclines/taxanes for locally recurrent and metastatic breast cancer. ${ }^{22}$

\section{Tumour growth is dependent on new \\ blood vessel formation, and inhibitors of angiogenesis are entering clinical trials as a combined adjuvant.}

A regimen of repetitive, low-dose chemotherapy and bevacizumab is effective against metastatic breast cancer, but metronomic chemotherapy alone is not (for example, low-dose oral cyclophosphamide).

There are potential problems with targeting downstream post-receptor signal transduction pathways. Complex interactions and 'cross-talk' exist, permitting compensation by adjacent pathways (functional redundancy) and disturbance of normal regulatory loops by therapeutic intervention. Inhibition of more distal steps, for example by mammalian target of rapamycin (mTOR) inhibitors, can result in reflexive activation of more proximal steps (e.g. Akt tyrosine kinase). Activity may be abrogated only by use of multiple inhibitors, and central signalling 'nodes' such as cyclin B1 are important and perhaps obligatory targets for sustained growth inhibitory effects. Animal models have shown promising responses from
Figure 3: Typical Speculated Appearance of a Carcinoma Seen on a Mammography

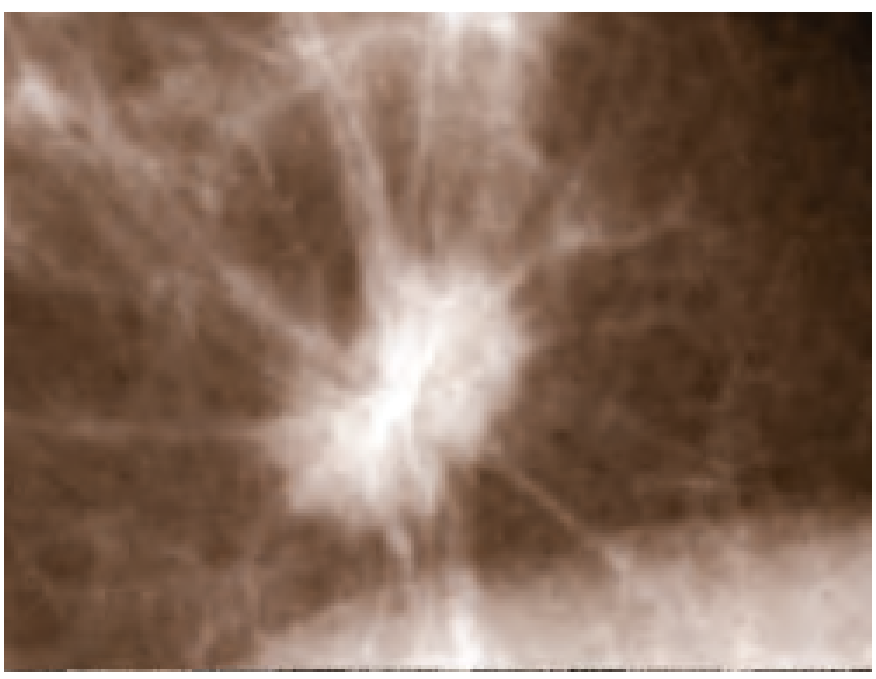

a combination of an aromatase inhibitor and the tyrosine kinase inhibitor gefitinib. This combination has not shown enhanced response rates compared with gefitinib alone in a pre-surgical schedule, although a greater reduction in rates of cell proliferation was evident for dual therapy. ${ }^{23}$ A phase III study of letrozole and lapatinib in metastatic breast cancer has shown a 30\% improvement in time to progression (10 versus 13 months; HR 0.769). Lapatinib inhibits all possible hetero-/homodimers of HER2/3 and should have superior efficacy to gefitinib. Trials are ongoing investigating this agent in combination with trastuzumab or letrozole as neo-adjuvant treatment. ${ }^{24}$

Farnesyl transferase inhibitors (FTIs) and mTOR inhibitors may be too far down the signal transduction pathway and do not possess a specific target. Some phase I/II studies have shown that mTOR inhibitors improve the efficacy of neo-adjuvant letrozole (CC1779) and possibly reverse resistance to this agent (RAD001). ${ }^{25}$ Other trials have been terminated early due to apparent increased cell survival and proliferation. Studies with FTls and letrozole have shown negative results to date.

Both antihormonal therapies acting via the $\mathrm{OR}$ and biological response modifiers targeting specific growth factor receptor pathways are capable of inducing mitogenic signals that allow breast cancer cells to survive initial systemic treatments and promote the emergence of a cohort of cells from which resistance develops. A cell's response to particular therapies must be anticipated and 'escape' mechanisms co-targeted. Short-term pre-surgical studies in the neo-adjuvant setting provide the opportunity to study proliferation indices before and after surgery, and changes in gene expression patterns may be predictive of response to novel agents and possibly of long-term outcomes.

\section{Aromatase Inhibitors}

Aromatase inhibitors represent the most significant advance in the endocrine management of breast cancer since the introduction of tamoxifen more than 30 years ago. Despite an established role as first- and second-line treatment for metastatic breast cancer, the optimal strategy for the incorporation of aromatase inhibitors into standard adjuvant endocrine schedules remains unclear. An upfront aromatase inhibitor might be indicated in those patients at higher risk of relapse (node-positive, ORpositive/PgR (progesterone recepetor)-negative, HER2-positive) for whom 
the amplitude of the hazard peak for recurrence is proportionately greater and could be suppressed or 'smoothed out' by an aromatase inhibitor more effectively than by tamoxifen. ${ }^{26,27}$ For those patients with lower hazards for relapse within the first two to three years, sequential therapy with tamoxifen followed by an aromatase inhibitor may be more appropriate and less costly. ${ }^{28}$ The absolute benefits of an aromatase inhibitor are very small in the first 36 months, during which period only $3.7 \%$ of patients relapse overall. Punglia et al. used a Markov analysis to develop models that simulated a 10-year disease-free survival among OR-positive women with early breast cancer. ${ }^{29}$ According to this analysis, switching from tamoxifen to an aromatase inhibitor after two to five years leads to a modest gain in diseasefree survival compared with monotherapy with an upfront aromatase inhibitor for five years. This analysis has been criticised on the basis of heterogeneity of end-points, with a 'dilutionary effect' of deaths without recurrence when disease-free survival is used, augmenting the relative benefits of switching. ${ }^{30} \mathrm{~A}$ variation of the Markov model, based on biological mechanisms, was used to demonstrate that when time to recurrence was taken as the primary end-point, upfront aromatase inhibitors were favourable. Outcome benefits must be balanced against long-term risks in terms of bone health and cognitive function. Any cost analysis must take account of the subsequent adverse events prevented (e.g. thromboembolism, gynaecological conditions). There are concerns about the impact of severe oestrogen deprivation in women receiving aromatase inhibitors for chemoprevention. The use of 'add-on' agents to minimise the complications of aromatase inhibitors (e.g. biphosphonates) detracts from a preventative strategy in the context of healthy women.

\section{Extended Adjuvant Endocrine Treatment}

Although more than three-quarters of recurrences occur within the first five years, late events do occur and patients remain at chronic risk of relapse.
Dormant cancer stem cells can be 'kick-started' many years after the primary treatment of breast cancer. Examination of hazard rates for recurrence within the extended adjuvant endocrine setting of the MA-17 trial implies that there is a residual risk of recurrence in patients completing five years of tamoxifen. The hazard ratio for recurrence shows a trend to decrease over time and there is greater benefit from letrozole with more prolonged therapy. ${ }^{31}$

\section{Genetic Profiling}

The sophisticated methods of genetic profiling with DNA microarrays and their integration with proteomics may ultimately yield both prognostic and predictive information and allow treatments to be better tailored to individuals. Human cancers display more molecular heterogeneity compared with animal models, and designated gene profiles must be rigorously validated against histopathological indices and independent data sets prior to any meaningful conclusions and assimilation into routine clinical practice. The '70 gene' profile divides untreated node-negative breast cancer patients into 'good' and 'poor' prognostic groups. ${ }^{32}$ This may reflect underlying paradigms of breast cancer biology, with the 'poor' group containing Fisherian-type tumours that are more likely to disseminate with the formation of distant metastases. There are unresolved issues relating to the stromal contribution to genetic profiles and the apparent absence of certain key genes involved in cell-cycle control. It is probably premature to consider these profiles sufficiently refined to guide clinical decision-making and permit an oncologist to confidently withhold chemotherapy when conventional parameters would favour such treatment. Bioinformatics will help decipher this new molecular information and encourage data to be constrained ('bin' rather than 'cluster'). In turn, such technology may fulfil its promise of matching patients and therapies. ${ }^{33}$
1. Fisher $B$, Laboratory and clinical research in breast cancer - A personal adventure: The David A Karnovsky Memorial Lecture, Cancer Res, 1980;40:3863-87.

2. Cady B, Stone MD, Schuler J, et al., The new era in breast cancer: invasion, size and nodal involvement dramatically decreasing as a result of mammographic screening, Arch Surg, 1996;131:301-8.

3. Hellman $S$, Natural history of small breast cancers, I Clin Oncol, 1994;12:2229-34.

4. Benson JR, Querci della Rovere $G$, The biological significance of ipsilateral local recurrence of breast cancer: determinant or indicator of poor prognosis, Lancet Oncology, 2002;345-9.

5. Baum $\mathrm{M}$, Does surgery accelerate the appearance of distant metastases? Conference on Ipsilateral Breast Cancer Recurrence, 2006.

6. Early Breast Cancer Trialists Collaborative Group (EBCTCG), Effects of radiotherapy and of differences in the extent of surgery for early breast cancer on local recurrence and 15-year survival: an overview of the randomised trials, Lancet, 2005;366:2087-2106.

7. Toth $B A$, Lappert $P$, Modified skin incision for mastectomy: the need for plastic surgical input in pre-operative planning, Plast Reconstr Surg, 1991;87:1048-53.

8. Benson JR, Skin-sparing mastectomy: Speciality bias and worldwide lack of consensus, Cancer, 2004;101:1099-1100

9. Rivadeniera DE, Simmons RM, Fish SK, et al., Skin-sparing mastectomy with immediate breast reconstruction: a critical analysis of local recurrence, Cancer I Sci Am, 2000;6:331-5.

10. Lyman GH, Guiliano AE, Somerfield MR, et al., The American Society of Clinical Oncology Guideline Recommendations for Sentinel Lymph Node Biopsy in Early Stage Breast Cancer, J Clin Oncol, 2005;23:7703-20.

11. Bleiweiss I, Sentinel lymph nodes in breast cancer after 10 years: rethinking basic principles, Lancet, 2006;7686-92.

12. Romrell $\mathrm{LJ}$, Bland $\mathrm{KI}$, Anatomy of the breast, axilla, chest wall and related metastatic sites. In: Bland KI, Copeland EM (eds),

\section{The Breast, 2004.}

13. MacMillan RD, Blamey RW, The case for axillary sampling, Advances in Breast Cancer, 2004;1:9-10.

14. Naik AM, Fey J, Gemignani M, et al., The risk of axillary relapse after sentinel lymph node biopsy for breast cancer is comparable with that of axillary lymph node dissection: followup study of 4,008 procedures, Ann Sur, 2004;240:462-8.

15. American College of Surgeons Oncology Group - ACSOG Z0011, A randomised study of axillary node dissection in women with clinical T1-2, NO, MO breast cancer who have a positive sentinel node, www.acosog.org/studies/organ

16. IBCSG 23-01 Protocol, www.ibcsg.org

17. Querci della Rovere G, Benson JR, Management of the axilla, Women Oncol Rev, 2006.

18. Schipper $H$, Baum M, Turley EA, Breast cancer: should we control rather than kill cancer cells? In: Calvo F, Crepin M,

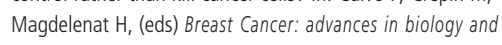
therapeutics, 1996:235-43.

19. Romond EH, Perez E, Bryant J, et al., Trastuzumab plus adjuvant chemotherapy for operable HER2-positive breast cancer, N Engl J Med, 2005;353:1673-84.

20. Rimawi MF, Mohsin K, Gutierrez MC, et al., Inhibiting the growth factor receptor (GFR) pathway preserves and enhances the expression of the estrogen receptor (ER) in HER-2/neu (HER2) overexpressing human breast tumors and xenografts. Breast Cancer Res Treat, 2005;94: abstract 8.

21. Kim W, Serrero G, PC-cell derived growth factor (PCDGF/GP88, progranulin) stimulates HER-2 phosphorylation and confers herceptin resistance to HER-2 overexpressing breast cancer cells, Breast Cancer Res Treat, 2005;94: abstract 10.

22. Miller $K$, Wang M, Gralow J, et al., A randomised phase III tria of paclitaxel versus paclitaxel plus bevacizumab as first-line therapy for locally recurrent or metastatic breast cancer: a trial co-ordinated by the Eastern Co-operative Oncology Group (E2100), Breast Cancer Res Treat, 2005;94: abstract 3.

23. Guix M, Kelley MC, Reyzer M, et al., Short course of EGFR tyrosine kinase inhibitor erlotinib (OSI-774, Tarceva) reduces tumour cell proliferation and active MAPK in situ in untreated operable breast cancers: strategy for patient selection into phase II trials with signalling inhibitors, Breast Cancer Res Treat, 2004;88:(Suppl. 1).

24. Osborne CK, Growth factor receptors and response, Conference on Controversies in Breast Cancer, 2006.

25. Awada A, Cardosa F, Fontaine C, et al., A phase lb study of the mTOR inhibitor RAD001 (everolimus) in combination with letrozole (Femara), investigating safety and pharmacokinetics in patients with advanced breast cancer stable or slowly progressing on letrozole, Breast Cancer Res Treat, 2004;88:1.

26. Baum M, ATAC update, Are there now sufficient data to replace tamoxifen with anastrozole as first-line therapy for hormone receptor positive post-menopausal breast cancer, 27th San Antonio Breast Cancer Symposium, 2004.

27. Howell A, ATAC trial update, Lancet, 2005;365:1225-6.

28. De Castro G, ATAC trial update, Lancet, 2005;365:1225.

29. Punglia RS, Kuntz KM, Winer E, et al., Optimising adjuvant endocrine therapy in post-menopausal women with early-stage breast cancers, J Clin Oncol, 2005;23:5178-87.

30. Cuzik J, Sasieni P, Howell A, et al., Should aromatase inhibitors be used as initial treatment or sequenced after tamoxifen, $\mathrm{Br}$ J Cancer, 2006;94:460-64.

31. Ingle JN, Goss PE, Tu D, et al., Analysis of duration of letrozole extended adjuvant therapy as measured by hazard ratios of disease recurrence over time for patients on NCIC CTG MA.17, Breast Cancer Res Treat, 2005;94:S11, abstract 17.

32. Van't Veer L, Dai H, van de Vijver MJ, et al., Gene expression profiling predicts clinical outcome of breast cancer, Nature, 2002;415:530-36.

33. Buyse $M$, Loi $S$, van't Veer $L$, et al., Validation and clinical utility of a 70-gene prognostic signature for women with nodenegative breast cancer, J Natl Cancer Inst, 2006;98:1183-92. 


\section{»Only MammaPrint can give you a clear answer in breast cancer prognosis.«}

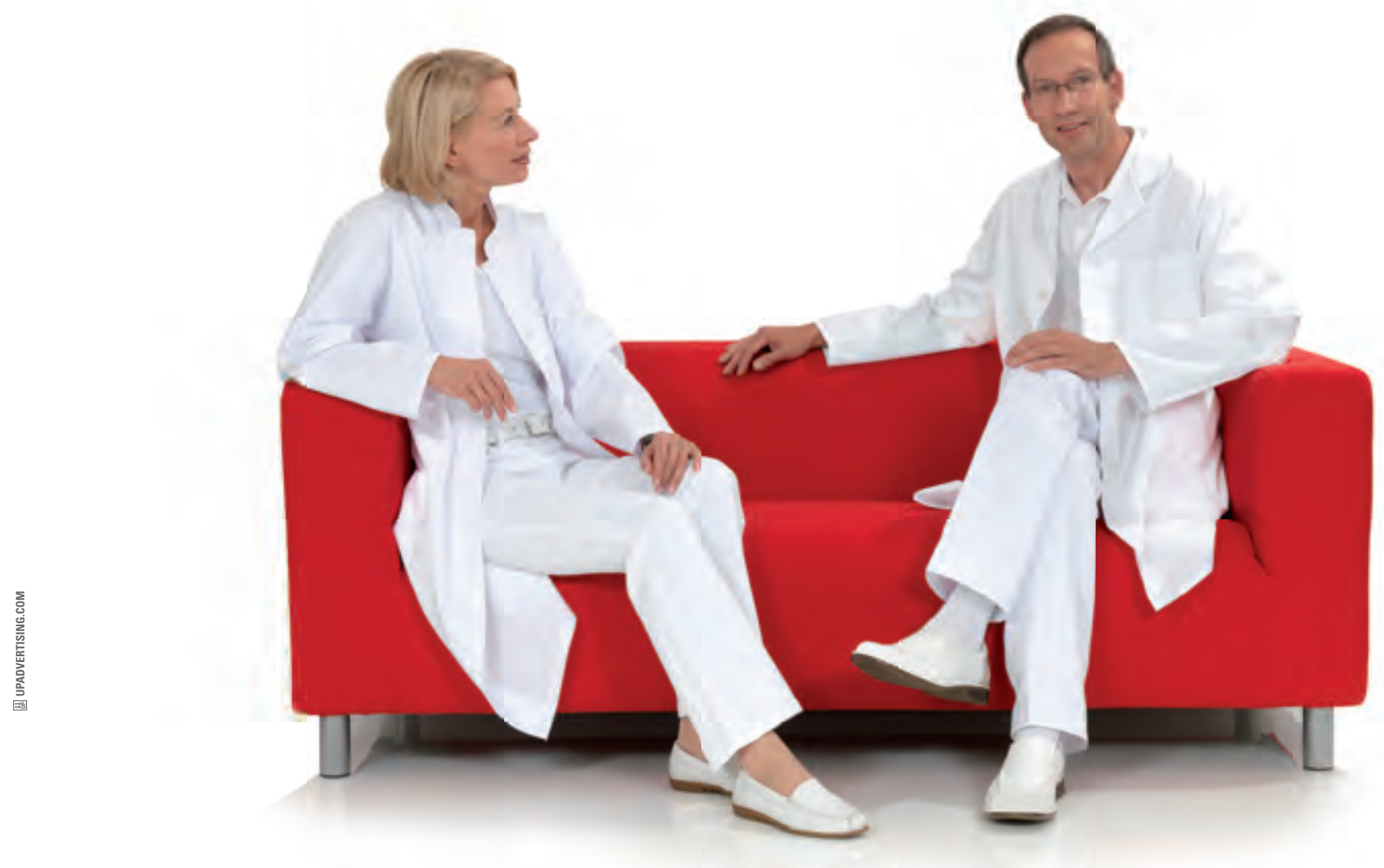

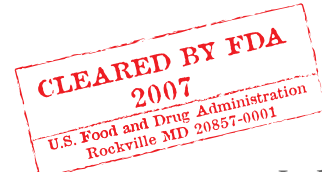

MammaPrint $^{\circledast}$ is the world's first in vitro Diagnostic Multivariate Index Assay cleared by FDA authorities. MammaPrint enables you to look inside every tumor at the 70 most informative genes identified from the whole genome. You receive clear low or high risk answers specific to the patient's tumor. MammaPrint has been validated in over 1,000 patients and, to date, more than 7,000 successful Please feel free to contact us for further MammaPrint tests have proven its clinical robustness. Simply collect a biopsy and prepare the MammaPrint Sampling Kit for shipment at room temperature. Reliable and fast delivery of your test results is our job.

MammaPrint is currently available for all breast cancer patients with up to three positive lymph nodes, ER+ or ER-, with no limitation in treatment. information or to order your MammaPrint test.

Phone: +31 205129161 or

email: customerservice@agendia.com.

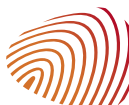

mammaprine" decoding breast cancer. 